REVIEW

\title{
Nutrition support for patients in the intensive care unit
}

\author{
R D Griffiths, T Bongers
}

Postgrad Med J 2005;81:629-636. doi: 10.1136/pgmj.2005.033399

Enteral nutrition (EN) is the mainstay of nutrition delivery within intensive care seeking to capitalise on its benefits for the gastrointestinal tract and associated immune system, but this has brought new challenges in delivery to the sick. The hoped for benefit has led to the mistaken belief by some that parenteral nutrition (PN) is no longer required. However, a greater appreciation of the risks of EN delivery in the sick patient combined with improvements in PN formulation and use help explain why PN is not as risky as some have believed. Real outcome benefits have been described with the new glutamine containing PN formulations. PN remains important in the presence of gastrointestinal feed intolerance or failure.

See end of article for authors' affiliations

Correspondence to: Professor R D Griffiths, Division of Metabolic and Cellular Medicine, School of Clinical Sciences, Duncan Building, UCD, Daulby Street, University of Liverpool, L69 3GA, UK; rdg@liverpool.ac.uk

Submitted 4 February 2005 Accepted 30 March 2005
$\mathrm{T}$ he intensive care unit (ICU) patient presents a number of nutritional challenges. The case mix of patients admitted to intensive care units may range from those admitted electively after major elective surgery to those admitted as emergencies after some surgical catastrophe, major trauma, sepsis, or respiratory failure. The variation in age range and prior health status may be extreme and nowadays ICUs are admitting increasingly more elderly, frail, or malnourished patients whose nutritional reserve may be severely compromised.

\section{RECOGNITION OF PRIOR NUTRITIONAL STATUS}

Many patients admitted in emergency may have been suffering an illness and have had poor nutrition before admission to intensive care. The best assessment of prior nutritional state is a detailed history of prior illness and nutritional intake combined with clinical examination of fat and muscle distribution. Body mass index (BMI $=$ weight in $\mathrm{kg} /$ height in $\mathrm{m}^{2}$ ) is useful but weight can be difficult to obtain accurately and may be distorted by resuscitative fluid administration. We know that ICU patients suffering from under-nutrition with a limited nutrition reserve have a poorer outcome ${ }^{1}$ and that having a low BMI has been shown to be an independent predictor of excess mortality in multiple organ failure. $^{2}$

WHAT IS OCCURRING METABOLICALLY? Increased metabolic requirements through increased protein breakdown and synthesis and changed substrate turnover occur through a range of demands depending on the various clinical situations. The enormous endocrine and cytokine flux of systemic inflammatory response common to sepsis or major trauma will increase basal metabolic rate usually proportional to the degree of insult and this is compounded by the effects of treatments such as adrenergic inotropes. However, what is not realised is that the total energy requirements may only modestly increase in the first few days. Heavy sedation and neuromuscular paralysis used to facilitate total ventilator support will reduce skeletal muscle activity. Detailed metabolic studies in ICU patients have shown that the total energy expenditure in the first week for patients with severe sepsis is $25 \mathrm{kcal} / \mathrm{kg} /$ day and for trauma patients $30 \mathrm{kcal} / \mathrm{kg} /$ day. What is often not appreciated is that by the second week evidence suggests this may have risen to as much as $40 \mathrm{kcal} / \mathrm{kg} /$ day in sepsis and perhaps even $55 \mathrm{kcal} / \mathrm{kg} / \mathrm{day}$ in some trauma cases. ${ }^{3}$ These are not necessarily nutrition targets but illustrate the wide variation in possible requirements.

What is more challenging to accept is how unreliable most predictive estimates of energy requirements are? Detailed metabolic measurements show wide patient variation between and within patients on different days. ${ }^{4}$ It has proved possible for uncomplicated surgery to suggest energy requirements of 1.0-1.15 times basal metabolic rate (BMR) while major surgery 1.25-1.4 $\times$ BMR is sufficient. Currently recommendations suggest that $25 \mathrm{kcal} / \mathrm{kg} /$ day $(105 \mathrm{~kJ} /$ $\mathrm{kg} /$ day) is a reasonable target intake for ICU patients initially for the first week however if too rigorously adhered to especially in some sepsis and trauma patients may be inadequate in the long run and a target of 30 or $35 \mathrm{kcal} / \mathrm{kg} /$ day (125-146 kJ/kg/day) may be more appropriate in subsequent weeks.

As long as over feeding is avoided to prevent excess lipid deposition the steady supply of protein is the most critical nutrient. Sequential studies on the ICU in severely septic patients with peritonitis ${ }^{5}$ show after an initial gain in body water with resuscitation, there is a large and progressive loss of protein despite full nutritional provision. Two thirds of the protein loss comes from skeletal muscle in the first 10 days, but later more is lost from the viscera. This loss of lean body mass (whole body water and protein) that ranges from $0.5 \%$ to $1.0 \%$ loss per day is far greater than that attributable to bed rest alone. This occurs in the context of full nutritional provision and not simple starvation and a consistent feature seen is that body fat could be preserved by adequate calorie provision. ${ }^{6}$ The rapidity and extent of the catabolic

Abbreviations: $\mathrm{EN}$, enteral nutrition; $\mathrm{PN}$, parental nutrition; ICU, intensive care unit; NJ, nasojejunal; NG, nasogastric; BMI, body mass index 
muscle wasting in the critically ill is astonishing with a reduction in muscle fibre cross sectional area of 3\%-4\% per day. ${ }^{7}$ In the first couple of weeks despite a 35\%-50\% decline in respiratory and skeletal muscle function there is no loss of cardiac mass or function in critically ill patients ${ }^{8}$ however wasting does occur with protracted illness.

Muscle wasting is ultimately a balance between protein synthesis and degradation. Various tissues and organs respond differently and change during the course of an illness. After modest surgery there is a decrease in whole body protein synthesis ${ }^{9}$ rather than breakdown. Short term starvation decreases skeletal muscle protein synthesis. ${ }^{10}$ With trauma and major surgery both synthesis and degradation increase, the latter being more enhanced..$^{11}$ In multiple organ failure increased whole body protein breakdown predominates over increased protein synthesis. ${ }^{12-14}$ To meet this metabolic demand for substrates involved in protein synthesis increased proteolysis occurs, particularly in skeletal muscle. This occurs through the energy demanding ubiquitin-proteasome pathway that requires ATP and is stimulated by fasting, acidosis, trauma, sepsis, and cancer. ${ }^{15}$ Glucocorticoids are important in this process not only in regulating and increasing the proteolysis in muscle but also increasing the utilisation of the resulting amino acids in the liver. A combination of insulin and essential amino acids oppose the catabolic effect mediated through mechanisms that inhibit proteolysis in the normal state. In the response to sepsis or injury the signal cascade of TNF and interleukins from activated macrophages and endothelial cells stimulate the ubiquitin-proteasome pathway in muscle probably involving multiple cytokine signals. Normally during prolonged starvation, after the initial mobilisation of amino acids for gluconeogenesis, protein breakdown decreases as increased energy is derived instead from fat metabolism. This important control preserving muscle protein is lost in the face of sustained stimulation of protein breakdown in sepsis and inflammation and endogenous glucose production continues. The release of amino acids during protein breakdown in skeletal muscle is not in proportion to the constituent amino acids, rather many are synthesised to produce glutamine that is released from muscle to meet the demand for additional amino groups and for added gluconeogenesis. ${ }^{16}$

The provision of high quality (essential and conditionally essential) amino acids along with insulin is central to good nutritional provision in the critically ill. As amino acids are not stored but used in synthesis or metabolised the provision needs to meet on going protein synthetic demand. Importantly, in contrast with the malnourished where protein synthesis can be stimulated by nutrition and catch up for missed feed is therefore possible, in the critically ill where synthesis may already be stimulated the ability to catch up for missed feeding is limited. Currently our best evidence in ICU based upon whole body measurements suggests at least $1.2-1.5 \mathrm{~g} / \mathrm{kg} /$ day of protein are needed. ${ }^{17}$

There are sound metabolic arguments for nutrition; the challenge comes in finding the safest and most reasonable way to deliver it because patients within intensive care except when recovering are unable to eat normally.

\section{SHOULD PATIENTS WITHIN INTENSIVE CARE BE FED ENTERALLY (EN) OR PARENTERAL (PN)?}

In the UK the past 15 years has seen a move away from providing nutrition support intravenously (parenteral nutrition, PN) to providing it nasogastrically or jejunally (enteral nutrition, EN). The reasons reflect changes in our understanding of nutrition and improved delivery systems. Indeed for some patients nowadays weaning off ventilator assistance may mean sitting out in chairs and taking an oral diet.
Key points 1

- Increased metabolic requirements arise from increased protein breakdown and synthesis and changed substrate turnover and vary depending on the clinical situation.

- There is wide patient variation between and within patients on different days.

- The catabolic depletion of protein reserves is one of the most striking features of the critically ill.

- The loss of protein has been shown to occur in the context of full provision of nutritional support and is not simply attributable to starvation.

The reduced use of PN is undoubtedly justified as many patients were given it in the past to their detriment ${ }^{18}$ despite still having a fully functional gastrointestinal tract. However, regarding modern PN practice compared with early EN the evidence firmly shows that after major surgery ${ }^{19} 20$ it is risk neutral overall. This is not to argue against EN as over the past decade it has been shown in the gastrointestinal surgical population that the overwhelming majority may be fed safely enterally ${ }^{21}$ but PN is still needed in $9 \%$ of these patients. One of the largest meta-analysis of 27 studies in 1828 surgical patients confirms that enteral feeding does carry a lower infective risk than PN RR 0.66 (95\% CI 0.56 to 0.79), but at the cost of a non-significant trend to increased complications RR 1.36 (95\% CI 0.96 to 1.83 ) and overall no advantageous effect on mortality risk RR 0.96 (95\% CI 0.55 to 1.65 ). ${ }^{22}$ They confirmed that a short term standard intravenous fluids regimen used in the normally nourished surgical patient has a lower risk of infection RR 0.77 (95\% CI 0.65 to 0.91 ) than the inappropriate early use of $\mathrm{PN}$ in these patients. In contrast in the malnourished patient unable to tolerate enteral feeding not giving parenteral feed significantly increases the mortality risk threefold, RR 3.0 (95\% CI 1.09 to 8.56). So a decision that results in the failure to give PN in a malnourished patient who cannot be fed enterally can increase the risk of death.

\section{WHAT IS THE EVIDENCE THAT SUPPORTS THE CURRENT DECISIONS REGARDING THE ROUTE OF NUTRITION TAKEN IN ICU?}

The underlying basis in favour of EN is that a failure to maintain normal oral nutrition is associated with immunological changes and impairment of the gut associated lymphatic system (GALT) that leads to the intestine, through lymphatic drainage, becoming the source of activated cells and proinflammatory stimulants during gut starvation. ${ }^{23}$ Other secondary abnormalities such as permeability changes and occasionally even bacterial translocations increase the immune challenge to the GALT but their contribution is probably secondary. This failure of immune defence was originally considered uniquely dependent on lack of enteral (luminal) nutrition. However, significant reversal of these defects has been shown in animal models using intravenous nutrition that specifically has added glutamine.

However, the evidence base that supports current best practice recommendations to encourage EN is not without controversy. A meta-analysis of early EN in "critically ill" surgical patients supported the view that it reduces the incidence of infection ${ }^{24}$ but evidence that real outcome is influenced is lacking. 15 studies of 753 patients showed a lower RR of 0.45 (95\% CI 0.3 to 0.66 ) for infection in favour of early enteral feeding with a reduction in length of stay of 
-2.2 days (95\% CI -0.81 to -3.63 ). However, nine of the studies were in patients after elective abdominal surgery indicating the term "critically ill" was used rather loosely. Furthermore, many studies also entailed the early application of feeding. This analysis is not helpful for general ICU practice because significant heterogeneity in the results meant not all studies or patients showed effects in the same direction and no conclusion could be made regarding medical intensive care patients or the amounts of feed. This case is revisited in the Canadian clinical practice guidelines for ventilated patients where they separated out the issue of PN and EN and also the issue to early compared with late introduction of the feed. ${ }^{25}$ With data limited to ICU patients there was no overall difference in mortality rates with either EN or PN with no apparent difference in mortality rates across groups receiving EN or PN (RR 1.08; 95\% CI 0.70 to 1.65). As suggested compared with PN, EN was associated with a significant reduction in infectious complications (RR $0.61 ; 95 \%$ CI 0.44 to $0.84 ; \mathrm{p}=0.003$ ). The early compared with late introduction of enteral feed only suggested that early EN was associated with a trend toward a reduction in mortality (RR $0.52 ; 95 \%$ CI 0.25 to $1.08 ; \mathrm{p}=0.08$ ) when compared with delayed nutrient intake and infection risk was not different. A duplicate systematic review publication of the EN compared with PN question, although presented in more detail, confirmed there was no difference in outcome, length of stay, days ventilation, or mortality ${ }^{26}$ dispelling the myth that PN if needed was harmful. A recent systematic review from Australia has moved the debate even further. In an attempt to overcome the criticism of including poor quality studies in reviews the authors have used a component based approach to investigate the effect of trial quality. ${ }^{27}$ The intention to treat analyses from nine studies comparing EN and PN were aggregated and showed a significant mortality benefit in favour of the use of PN (odds ratio, OR 0.51, 95\% CI 0.27 to $0.97, \mathrm{p}=0.04$ ) confirming what many commentators have been saying. ${ }^{28}$ This difference was lost when only those studies (and therefore a selected population of patients) where early enteral feeding was used. The risk of infection with PN remained but it must be noted that this risk is insufficient to affect outcome.

Great care should be taken not to over interpret these reviews either way. The most important fact was that in all these studies no consideration was taken regarding the functional state of the gastrointestinal tract as patients could be randomised to either route of feeding.

\section{Key points 2}

- Enteral nutrition (EN) as the first choice, is usually delivered nasogastrically and is justified on cost alone.

- Many patients were given parenteral nutrition (PN) in the past despite having a fully functional gastrointestinal tract. However, PN is still needed in $9 \%$ of these patients so cannot be forgotten.

- EN does carry a lower infective risk than PN, RR 0.66 (95\% Cl 0.56 to 0.79 ), but at the cost of a nonsignificant trend to increased complications RR 1.36 (95\% Cl 0.96 to 1.83 ).

- Meta-analysis shows that EN compared with PN is either risk neutral for mortality risk RR $0.96195 \% \mathrm{Cl}$ 0.55 to 1.65 ) or PN may even offer a lower risk 0.51 (95\% Cl 0.27 to 0.97 )

\section{WHEN GASTROINTESTINAL FUNCTION IS IN DOUBT MIGHT PN EVEN BE SAFER THAN EN?}

The only level 1 study that has addressed the EN compared with PN question of benefit to ICU doctors is a fascinating "pragmatic" study by John MacFie's group in Scarborough, England. ${ }^{29}$ The study looked at the clinical outcomes of all 562 patients given EN and PN in a major hospital under the auspices of a specialist gastroenterology nutrition service. EN was given to 237 patients with a functioning gastrointestinal tract; PN to 267 with absolute gastrointestinal failure; and this left a group of 64 where it was unclear if the gastrointestinal function was adequate. They randomised this last cohort of patients to compare EN and PN. They therefore made sure that the risks were only applied to those that need them and overcame the problems of all previous comparisons of EN and PN where opinions might differ as to the appropriateness of the particular choice of route of feeding.

There was obviously a noticeable difference in the patients and the diseases in the first two non-randomised groups that received either PN or EN. Strikingly there was no difference in septic morbidity but a higher non-septic complication rate in the enteral feeding group. These complications were not minor and significant excess in mortality was seen with EN emphasising some of the mortality risks associated with EN. In the non-randomised groups one might think this increased mortality in the EN patients could be explained by the difference in patient population was it not that the exact same relation with higher mortality was seen in the group of patients where randomisation had occurred. Inadequate nutritional intake characterised patients receiving EN. Inclusion of these important data in the most recent meta-analysis contributes to the evidence that the risk of enteral feeding on mortality may even exceed that of PN.

\section{EN DELIVERY PROBLEMS AND ASPIRATION}

To understand the debate however one must appreciate the issue of delivery and risk of aspiration for ventilated intensive care patients as the sicker ones have increasing gastrointestinal intolerance. A recent study in France of 153 nasogastrically fed patients showed that upper digestive intolerance is a frequent event in the critically ill and associated with pneumonia $(43 \% \vee 24 \% \mathrm{p}=0.01)$, longer ICU stay (23v 15 days $\mathrm{p}=0.007)$, and increased mortality (41\% v 25\% $\mathrm{p}=0.03) .{ }^{30}$ Even corrected for illness severity the risk of death was significantly increased RR 1.48 (95\% CI 1.04 to 2.10). This increased risk is an important issue that gets buried in many enteral studies that involve broad populations of patients with different illness severity. The risks for intolerance were not surprising because of sedation use (RR $1.7895 \%$ CI 1.17 to 2.71 ) and catecholamine use (RR 1.81, $95 \%$ CI 1.21 to 2.70 ) both features of genuine intensive care patients, particularly those with sepsis and shock. The evidence that using motility agents or nasojejunal (NJ) feeding rather than nasogastric (NG) feeding significantly changes these risks is lacking. A meta-analysis found that the evidence that motility agents can affect any aspect of outcome was wanting. ${ }^{31}$ A multicentre study from Spain confirms that $\mathrm{NJ}$ feeding does not reduce the incidence of pneumonia. ${ }^{32}$ In 101 randomised ICU patients there was no difference in feeding duration, length of stay, or mortality (NG 43\% v NJ 38\%). Although the NJ group had lower gastrointenstinal complications there was a similar incidence of nosocomial pneumonia (NG 40\% v NJ 32\%). While a study from Melbourne, Australia ${ }^{33}$ suggested improved tolerance with NJ feeding and a low requirement for PN another study in 60 USA medical patients showed that NG fed patients reached their target goal earlier. ${ }^{34}$ There was no difference in aspiration events. These studies are consistent with an earlier 
study of 44 patients similarly randomised where small bowel feeding led to a significant increased proportion of the nutrition target achieved $(p=0.05)$ but definitely no reduction in ventilator pneumonia where the risk with small bowel feeding was RR 1.1 (95\% CI 0.96 to 2.44). ${ }^{35}$ A rare but often fatal complication of non-occlusive bowel necrosis has been reported in critically ill trauma patients fed into the small bowel. ${ }^{36}$ As this serious complication cannot be detected early there is no overwhelming risk free evidence supporting $\mathrm{NJ}$ feeding in preference to NG feeding for the reduction in infective risk.

\section{DOES IT MATTER THAT ENTERAL FEEDING MAY NOT DELIVER SUFFICIENT?}

Several surveys have shown that the real practice in intensive care is to deliver considerably less than that prescribed ranging between $50 \%$ and $60 \%$ of target. ${ }^{37-39}$ What is "sufficient" is open to debate and there are some advocates of deliberate underfeeding. However, underfeeding is a concern as it has been suggested that medical ICU patients who received less than $25 \%$ of target feed have a higher risk of nosocomial blood stream infections. ${ }^{40}$

Feeding earlier should increase delivery but a study on early feeding in medical patients ${ }^{41}$ succeeded only in showing that poor nutrition support is common. They studied 150 ventilated, medical ICU patients, in a prospective but unblinded study using unfortunately consecutive rather than random allocation. The patients were fed using the NG or NJ method following a protocol to reach either a target of $25 \mathrm{kcal} / \mathrm{kg}$ on day $\mathrm{l}$ (early feeders) or only $20 \%$ of the target until day 5 (late feeders). In contrast with discussion earlier on surgical patients the 75 patients who were early fed had a significantly $(p=0.02)$ higher incidence of ventilator associated pneumonia $49.3 \%$ compared with $30.7 \%$. Not surprisingly the early feeders had a longer ICU stay of 13.6 compared with 9.8 days $(p=0.04)$. Close inspection however shows that both groups received little nutrition with the percentage of target achieved in the early feeders of only $28 \%$ and even less at $7 \%$ in the late feeders $(p=0.001)$. Despite having a higher incidence of ventilator pneumonia and longer ICU stay the early feeders had a non-significant trend to lower hospital mortality $20.7 \%$ compared with $26.7 \%$ arguing against the view to semi-starve ICU patients. It could be argued that it was the increased risk of aspiration in the early feeding group that increased their morbidity, but that in the long run the greater nutrition improved their outcome?

Currently the most reasonable approach is to feed to meet the patient's energy expenditure with care to avoid overfeeding. With PN overfeeding is far easier while as shown with EN it is virtually impossible. The introduction of guidelines encouraging increased nutrition prescription and use of enteral feeding, often starting earlier has increased nutrition prescription..$^{42}$ Many ICUs follow nutrition protocols that use gastric residue as the measure of tolerance. While these protocols do encourage consistent delivery care must be

\section{Key points 3}

- In the malnourished patient unable to tolerate enteral feeding not giving parenteral feed significantly increases the mortality risk threefold, RR $3.0195 \% \mathrm{Cl}$ 1.09 to 8.56 ).

- The degree of underfeeding is a concern as it has been suggested that medical ICU patients who received less than $25 \%$ of target feed have a higher risk of nosocomial blood stream infections. taken not to over interpret single gastric residual volumes too rigorously without regard to the overall patient condition, progress of feeding, and other signs such as abdominal bloating. Aspiration risk remains the greatest concern but in ventilated ICU patients residual volumes are a poor marker of aspiration risk with the same incidence whether targets of 150 or $400 \mathrm{ml}$ are set. ${ }^{43}$ This is because aspiration can occur during many nursing procedures and turning and it is unclear how much the complication of pneumonia is related to direct feed aspiration (incidence 22\% range 0\%-94\%) rather than changed pharyngeal colonisation secondary to feed regurgitation (incidence $31 \%$ range $0 \%-94 \%$ ). ${ }^{43}$

Nevertheless there is a suggestion that guidelines may improve outcome. A 14 hospital cluster study from Canada, the ACCEPT study, showed that survival from intensive care was improved when an evidence based guideline for nutrition was followed and more nutrition was delivered more consistently. This was achieved by earlier introduction and more complete EN delivery without any decline in the use of PN alone or in supplementation. ${ }^{44}$ This result is astonishing particularly when tested through a cluster study that involves behaviour change. It may well be with greater dissemination of these guidelines attempts to replicate these findings in larger studies will not be successful. Nevertheless the data suggest that more is better than less confirming that poor nutrition intake is a problem.

\section{PN ADDED AS A SUPPLEMENT TO EN}

What to do if EN is failing to deliver? Is there evidence that supplementing EN with PN brings benefit or does it just add a new risk? This can take two approaches, where EN and PN are started concurrently and the PN weaned down as the patient becomes more EN tolerant or where EN is started and PN introduced only after some days have confirmed that the patient is intolerant of EN. Sadly only the former has been explored in research studies and represents a weakness in our data as the latter approach firmly tests the integrity of the gastrointestinal tract and avoids over feeding with PN in the initial period. In a well designed randomised, controlled study in a French mixed general ICU population $(n=120)$ it was shown that achieving a higher nutrition intake with parenteral supplements led to a faster recovery of plasma protein markers, their primary end point. They also showed a reduction in length of hospital stay of 2.5 days but it is doubtful if the power of the study was sufficient to convincingly prove this. ${ }^{45}$ All other measures including infective morbidity showed no significant difference. These findings were in a well nourished population (only 9\% were severely malnourished) receiving supplementation for up to seven days. More impressive benefits may have been shown if this study had investigated a higher risk group with preexisting malnutrition or reduced intake before ICU and they had supplemented for longer. The extent of nutrient deficit in this study was probably insufficient to affect outcome greatly.

\section{Key points 4}

- Survival from intensive care was improved when an evidence based guideline for nutrition was followed and more nutrition was delivered more consistently. This was achieved by earlier introduction and more complete EN delivery without any decline in the use of $\mathrm{PN}$ alone or in supplementation.

- One of the real landmark outcome studies in recent years showed the great benefits of glycaemic control in patients receiving early enteral or parenteral nutrition 
The study confirmed there was no evidence that the addition of PN added any risks or caused harm. A resent meta-analysis suggested there is no evidence that supplemental PN is a risk in ICU as long as overfeeding is avoided but starting it early and concurrently in the non-malnourished is not yet warranted. ${ }^{46}$ Whether starting PN early in those patients unable to start early enteral feeding is beneficial as hypothesised by the recent Australian review is speculative and needs to be formally tested. ${ }^{27}$

Perhaps what is more important is to take a serious approach to nutrition and metabolic control overall. One of the real landmark outcome studies in recent years showed the great benefits of glycaemic control in patients receiving early EN or $\mathrm{PN}^{.47}$ Closely applied best evidence nutrition protocols were central to this process. The long term outcome was related to the tight glycaemic control achieved with insulin, and not by underfeeding. We know that taking a different approach and underfeeding by omitting lipids and delivering hypocaloric parenteral feeds neither prevents hyperglycaemia nor its infectious complications. ${ }^{48}$

\section{WHY IS THERE PERHAPS LESS RISK WITH MODERN PN WITHIN INTENSIVE CARE PRACTICE?}

Reflecting on its origins and the state of nutrition support in the 1960s by just one of the pioneers will enlighten those interested on how far we have come. ${ }^{49}$ Such considerable experience over the past decades has highlighted the potential clinical complications of TPN and established the practical guidelines to minimise their impact. As the risks of indiscriminate and inappropriate use of PN are recognised they can be prevented by not overfeeding, and conscientious monitoring. Careful sterile line placement will minimise infections and some of the immunosuppression can be attenuated by supplementation of glutamine. Carefully respecting guidelines will go a long way to reduce the rate of complication but lack of consistent practice means that audit studies still regularly show over or underfeeding and shortcomings in PN prescription. ${ }^{50}$ It is therefore not surprising that some people carry a misguided interpretation that PN in itself rather than how it is used or misused in intensive care carries an excess mortality in patients where $\mathrm{EN}$ is difficult or impossible. It should not be forgotten that intensive care is potentially one of the safest environments for risky procedures as the high staff presence and experience

\section{Nutrition in the ICU: simple rules}

- Feed the malnourished and plan for those soon to be

- Start some enteral feeding if safe and as soon as practical.

- Use simple standard complete regimens, decisions over volumes of fluid dominate.

- Modest targets based on patient size bands are easier to achieve $(25 \mathrm{kcal} / \mathrm{kg} /$ day with $1.5 \mathrm{~g}$ protein $/ \mathrm{kg} /$ day).

- Use protocols, monitor delivery, note deficits, and act to meet targets.

- Use parenteral to complement or replace if delivery fails.

- Give sufficient insulin for glycaemic control using established protocols.

- Do not exacerbate glutamine deficiency, include in all PN preparations.

- Don't miss feeding because it is harder to catch up.
Key references

- Reid CL. Nutritional requirements of surgical and critically-ill patients: do we really know what they need? Proc Nutr Soc 2004;63:467-72

- Braunschweig CL, Levy P, Sheean PM, et al. Enteral compared with parenteral nutrition: a meta-analysis. Am J Clin Nutr 2001,74:534-42

- Martin CM, Doig GS, Heyland DK, et al, for the Southwestern Ontario Critical Care Research Network. Multicentre, cluster-randomized clinical trial of algorithms for critical-care enteral and parenteral therapy (ACCEPT). CMAJ 2004; 170:197-204

- Van den Berghe G, Wouters P, Weekers F, et al. Intensive insulin therapy in the critically patient. $N$ Engl J Med 2001;345:1359-67

- Griffiths RD Specialised nutrition support in critically ill patients. Curr Opin Crit Care 2003;9:249-59

should anticipate and prevent complications. For instance line infections have a serious association with mortality outside intensive care but recent evidence from intensive care shows that nowadays it is no longer possible to show an attributable causal link between intravenous catheter line infections and mortality. ${ }^{51}{ }^{52}$ This is presumably because our practice of line management is greatly improved and any infective line is only a symptom of the patient's illness and not a significant independent contributor to mortality. The relation between sepsis and nutrition in ICU and how it affects outcome has recently been reviewed..$^{53}$

The progressive development in formulation has improved PN used in the critically ill. The debate that intravenous fat emulsions have a detrimental effect on lung function in sepsis has been explored and shown that when infused at the normal recommended rates used in continuous all-in-one formulations to have no deleterious effect in septic patients. ${ }^{54}$ It seems it requires excessively rapid infusions inconsistent with good clinical practice to show any harmful effect. This is a good argument why all-in-one PN preparations should be universally available to intensive care.

The most significant formulation change in recent years to have entered ICU practice is the inclusion of glutamine to overcome a conditional deficiency. ${ }^{16}$ This reflects different requirements in the critically ill that are not met by conventional formulations that had compromises made for pharmaceutical reasons. Existing formulations that may be adequate to maintain a patient long term while receiving home PN have been proved inadequate for the changed requirements of the critically ill. The first double blind randomised outcome study comparing a standard PN with a glutamine enhanced PN within intensive care patients with gut failure as part of multi-organ failure ${ }^{55}$ showed a significantly improved six month survival ( 24 of 42 compared with 14 of $42, p=0.049$ ). Subsequent data showed that glutamine recipients have a significantly lower incidence of catheter related infections $(p=0.026),{ }^{56}$ but overall only a non-significant and modest reduction in early acquired infections as the opportunity for new infections is so high in these patients. More importantly though there was a later reduction in infection and the survival of patients with candida infections was improved. There was a clear link between duration of glutamine and survival with a reduced intensive care mortality from multiple organ failure in those patients remaining in ICU for a longer period and requiring a minimum of five days of parenteral feed $(p=0.05)$ and a 
median feed duration of 15 days. Meta-analysis supports this finding $^{57}$ and another recent study from Germany confirms improved outcome ${ }^{58}$ and is now a recommended standard. ${ }^{25}$

\section{IS THERE A PLACE FOR ENTERAL IMMUNE NUTRITION COCKTAILS TO MODIFY INFLAMMATION WITHIN ICU?}

It should not be forgotten that nutrition delivery in ICU is on the background of a changing and evolving pathophysiological state. A feature of most patients is an evolving inflammatory response where the decision to start nutrition may not arise at the same time in the process. The importance of the inflammatory response to activate defence mechanisms against infection, to direct cell movement, and induce endogenous cytoprotective mechanisms must be balanced by the collateral damage that occurs to various tissues.

Some studies have comprised single additional substrates such as glutamine or ornithine-alpha-keto-glutarate while other formulations include a fixed dose mixture of substrates. The evidence base in humans for the various additional substrates is variable and for the more complex mixtures at the fixed doses used within these formulations or "cocktails" is simply lacking.

\section{IMMUNE NUTRIENT COCKTAILS}

Apart from one unblinded study using an immune nutrient cocktail that showed an improved ICU survival in septic patients $^{59}$ evidence for benefit is lacking. Taking the two largest studies $^{60}{ }^{61}$ that contain hospital mortality data collectively the same immune nutrient feed produces a significant excess mortality OR 1.45 (CI 1.06 to 2.11) $\mathrm{p}=0.02$. This is disturbing because it suggests that more harm than good may occur. As benefits were said to occur in sub-sets of patients, it could be that some patients might benefit while others suffer harm. Mortality risk and benefits should be looked at using the decision to feed (intention to treat) as one does in practice.

A number of systematic reviews have tried to put sense to the poor evidence but given the heterogeneity of the feeds and the patients studied they provide little direct clinical guidance than the various studies individually. The most detailed meta-analysis ${ }^{62}$ suggests benefit seems to be in nonintensive care perioperative nutrition where especially prefeeding alone before the stress event significantly reduces infective complications and morbidity resulting in reduced length of stay. ${ }^{63}$ This clearly has limited relevance for most intensive care admissions but does underline an important principle of effect. More worrying is that within the possible intensive care patient populations that have been studied considerable heterogeneity exists in the results and together with this a suggestion of an increased mortality risk that was hidden within the presentation format of the original studies. It is suggested that the universal use of immune nutrient feed cocktails of mixed formulations cannot be recommended within intensive care at present and they should be confined to the specific populations where clinical benefit has been shown..$^{64}$

\section{CAN ENTERAL FEED MODIFY OR WORSEN SPECIFIC DISEASE PROCESSES?}

From a background of detailed animal work a novel enteral feed containing a changed lipid profile and increased antioxidants moderated the severity of the initial inflammatory response in the lungs of patients developing acute respiratory distress syndrome (ARDS). ${ }^{65}$ Feeds with increased antioxidants alone have shown little discernible benefit. ${ }^{66}$ The combination of eicosapentaenoic acid (EPA; fish oil), $\gamma$ linolenic acid (GLA; borage oil) had been shown to modulate membrane phospholipids composition with a shift towards a less inflammatory eicosanoid pattern.

Patients were recruited with firm pathological diagnosis, bronchoalveolar lavage was used to show a reduction in lung inflammation, and outcome measures showed improved parameters of gas exchange, reduction in duration of ventilation, decreased ICU stay, and decreased new organ failures. Subsequent analysis showed that the feed reduced the alveolar inflammatory response and their mediators to account for the improvements in gas exchange. ${ }^{67}$ However, the control group received a commercial high n- 6 fat feed and not necessarily a standard ICU enteral feed. We do not know how applicable these results are to patients with more severe ARDS than in the study group or those early at risk of ARDS? The underlying genetic status of the patients may not only determine the severity of their ARDS but also their response to lipid modification.

In recent years small animal models have re-examined the appropriateness of enteral feeding in the very ill and septic subject. In these EN was associated with a worse mortality and was less effective at augmenting muscle and liver protein synthesis compared with PN. ${ }^{68}$ Interim analysis of a recent randomised multicentre study in Italy showed that the ICU mortality of patients with severe sepsis given EN was significantly higher than those for PN $(44 \% \quad v \quad 14.3 \%$ $\mathrm{p}=0.039){ }^{68}$

\section{CONCLUSION}

A number of simple nutrition rules are clear. ICU patients staying more than a few days will need nutrition support and if malnourished they may need it sooner. Enteral feeding should be encouraged using simple feeding protocols and started early if safe to do so. However, it should not be forced if gastrointestinal intolerance is evident. We should use PN cautiously and follow guidelines of best practice. It is too easy to overfeed. It is reserved for those with verified gastrointestinal intolerance-that is, either in the context of gastrointestinal failure for total $\mathrm{PN}$ or gastrointestinal intolerance for supplemental PN. There is at present no evidence for "early" PN. We should ensure tight glycaemic control with insulin and use glutamine containing PN formulations in all-in-one mixtures.

\section{MULTIPLE CHOICE QUESTIONS (TRUE (T)/FALSE (F); ANSWERS AT END OF REFERENCES)}

A. Regarding nutritional status and nutritional requirements

(1) The nutritional status has an effect on outcome (morbidity and mortality) in the critically ill.

(2) If critically ill patients are severely under fed $(<25 \%$ of target feed) they have a higher risk of nosocomial blood stream infections.

(3) All patients on intensive care have a similar energy expenditures of about $25 \mathrm{kcal} / \mathrm{kg} /$ day through their illness and are therefore fed using the same feeding regimen.

(4) Over feeding is not a problem with patients on intensive care as the energy requirements are significantly increased.

(5) Protein loss does not normally occur in critically ill patients and if present suggests starvation.

B. What is regarded as "best" nutrition in the critically ill and how should it be delivered?

(1) Similar to starvation protein breakdown decreases when energy requirements are increasingly met by fat metabolism. 
(2) Increased proteolysis occurs (particularly skeletal muscle) in the critically ill to meet the increased metabolic demand for protein synthesis.

(3) Provision of high quality (essential and conditionally essential) amino acids along with insulin is central to good nutrition as amino acids are not stored but used in synthesis or metabolised during critical illness.

(4) Enteral feeding carries a lower risk of infections and is therefore superior to parenteral nutrition.

(5) Despite improved delivery systems and better quality of enteral feed there is still a number of patients who require parenteral nutrition because of gastrointestinal failure.

\section{Regarding enteral nutrition use in the critically ill}

(1) Upper digestive intolerance is a frequent event in the nasogastrically fed critically ill patient and is associated with pneumonia, longer ICU stay, and increased mortality.

(2) Upper digestive intolerance increases with sedation and catecholamine use.

(3) Nasogastric feeding can cause non-occlusive bowel necrosis, a life threatening complication.

(4) Nasojejunal feeding is associated with fewer aspiration complications compared with nasogastric feeding.

(5) Several surveys suggest, that under feeding is common practice on intensive care when feeding enterally.

\section{Further aspects of nutrition in intensive care}

(1) Survival from intensive care improves when an evidence based guideline for nutrition is followed.

(2) Glycaemic control in patients receiving early enteral or parenteral nutrition in mandatory.

(3) There is good evidence supporting the use of immune nutrient cocktails in intensive care.

(4) A novel enteral feed containing a combination of eicosapentaenoic acid (EPA; fish oil) and $\gamma$-linolenic acid (GLA; borage oil) shows a reduction in lung inflammation.

(5) Strong evidence suggests that including glutamine within parenteral nutrition is preferred in the critically ill.

\section{Authors' affiliations \\ R D Griffiths, T Bongers, Intensive Care Research Group, Division of} Metabolic and Cellular Medicine, School of Clinical Sciences, University of Liverpool, and Intensive Care Unit, Whiston Hospital, Merseyside, UK

Funding: none.

Conflicts of interest: none.

\section{REFERENCES}

1 Giner M, Laviano A, Meguid MM, et al. In 1995 a correlation between malnutrition and poor outcome in critically ill patients still exists. Nutrition 1996; 12:23-9.

2 Galanos AN, Pieper CF, Kussin PS, et al. Relationship of body mass index to subsequent mortality among seriously ill hospitalized patients. SUPPORT Investigators. The study to understand prognoses and preferences for outcome and risks of treatments. Crit Care Med 1997;25:1962-8.

3 Uehara M, Plank LD, Hill GL. Components of energy expenditure in patients with severe sepsis and major trauma: a basis for clinical care. Crit Care Med 1999;27:1295-302.

4 Reid CL. Nutritional requirements of surgical and critically-ill patients: do we really know what they need? Proc Nutr Soc 2004;63:467-72.

5 Plank LD, Connolly AB, Hill GL. Sequential changes in the metabolic response in severely septic patients during the first 23 days after the onset of peritonitis. Ann Surg 1998;228:146-58.
6 Streat SJ, Beddoe AH, Hill GL. Aggressive nutritional support does not prevent protein loss despite fat gain in septic intensive care patients. J Trauma 1987;27:262-6.

7 Helliwell TR, Wilkinson A, Griffiths RD, et al. Muscle fibre atrophy in patients with multiple organ failure is associated with the loss of myosin filaments and the presence of lysosomal enzymes and ubiquitin. Neuropathol Appl Neurobiol 1998;24:507-17.

8 Hill AA, Plank LD, Finn PJ, et al. Massive Nitrogen loss in critical surgical illness. Effect on cardiac mass and function. Ann Surg 1997;226:191-7.

9 Crane CW, Picou D, Smith R, et al. Protein turnover in patients before and after elective orthopaedic operations. Br J Surg 1977;64:129-33.

10 Essen P, McNurlan MA, Wernerman J, et al. Short term starvation decreases skeletal muscle protein synthesis rate in man. Clin Physiol 1992;12:287-99.

11 Birkhahn RH, Long CL, Fitkin D, et al. Effects of major skeletal trauma on whole body tunrover in man measured by L-(1, 14C)-leucine. Surgery 1980;88:294-300.

12 Arnold J, Campbell IT, Samuels TA, et al. Increased whole body protein breakdown predominates over increased whole body protein synthesis in multiple organ failure. Clin Sci 1993;84:655-61.

13 Essen P, McNurlan MA, Gamrin L, et al. Tissue protein synthesis rates in critically ill patients. Crit Care Med 1998;26:92-100.

14 Wernerman J, Hammarqvist F, Gamrin L, et al. Protein metabolism in critical illness. Baillieres Clin Endocrinol Metab 1996;10:603-15.

15 Mitch WE, Goldberg AL. Mechanisms of muscle wasting: the role of the ubiquitin-proteasome pathway. N Engl J Med 1996;335:1897-905.

16 Griffiths RD. The evidence for glutamine use in the critically-ill. Proc Nutr Soc 2001;60:1-8

17 Ishibashi N, Plank LD, Sando K, et al. Optimal protein requirements during the first 2 weeks after the onset of critical illness. Crit Care Med 1998;26:1529-35.

18 Heyland DK, MacDonald S, Keefe L, et al. Total parenteral nutrition in the critically ill patient: a meta-analysis. JAMA 1998;280:2013-19.

19 Braga M, Gianotti L, Gentilini O, et al. Early postoperative enteral nutrition improves gut oxygenation and reduces costs compared with total parenteral nutrition. Crit Care Med 2001;29:242-8.

20 Pacelli F, Bossalo M, Papa V, et al. Enteral vs parenteral nutrition after major abdominal surgery: an even match. Arch Surg 2001;136:933-6.

21 Braga M, Giannotti L, Gentilini O, et al. Feeding the gut early after digestive surgery: results of a nine-year experience. Clin Nutr 2002;21:59-65.

22 Braunschweig CL, Levy $\mathrm{P}$, Sheean PM, et al. Enteral compared with parenteral nutrition: a meta-analysis. Am J Clin Nutr 2001;74:534-42

23 Kudsk KA. Effect of route and type of nutrition on intestine-derived inflammatory responses. Am J Surg 2003;185:16-21.

24 Marik PE, Zaloga GP. Early enteral nutrition in acutely ill patients: a systematic review. Crit Care Med $2001 ; 29: 2264-70$.

25 Heyland DK, Dhaliwal RD, Drover JW, et al. Canadian clinical practice guidelines for nutrition support in mechanically ventilated, critically ill adult patients. JPEN J Parenter Enteral Nutr 2003;27:355-73.

26 Gramlich L, Kichian K, Pinilla J, et al. Does enteral nutrition compared to parenteral nutrition result in better outcomes in critically ill adualt patients? A systematic review of the literature. Nutrition 2004;20:843-8.

27 Simpson F, Doig GS. Parenteral vs. enteral nutrition in the critically ill patient: a meta-analysis of trials using the intention to treat principle, Intens Care Med 2004; epub 10.1007/200134-004-2511-2

28 Griffiths RD. Is parenteral nutrition really that risky in the intensive care unit? Curr Opin Clin Nutr Metab Care 2004;7:175-81.

29 Woodcock NP, Zeigler D, Palmer D, et al. Enteral versus parenteral nutrition: a pragmatic study. Nutrition 2001;17:1-12.

30 Mentec H, Dupont H, Bocchetti $M$, et al. Upper digestive intolerance during enteral nutrition in critically ill patients: frequency, risk factors, and complications. Crit Care Med 2001;29:1955-61.

31 Booth CM, Heyland DK, Parterson WG. Gastrointestinal promotility drugs in the critical care setting: a systematic review of the evidence. Crit Care Med 2002;30: 1429-35.

32 Montejo JC, Grau T, Acosta J, for the Nutritional and Metabolic working group of the Spanish Society of Intensive Care Medicine and Coronary Care Units, et al. Multicenter, prospective, randomized, single-blind study comparing the efficacy and gastrointestinal complications of early jejunal feeding with early gastric feeding in critically ill patients. Crit Care Med 2002;30:796-800.

33 Davies AR, Froomes PR, French CJ, et al. Randomised comparison of nasojejunal and nasogastric feeding in critically ill patients. Crt Care Med 2002;30:586-90

34 Neumann DA, Delegge MH. Gastric versus small-bowel tube feeding in intensive care unit: a prospective comparison of efficacy. Crit Care Med 2002;30: 1436-8

35 Kearns PJ, Chin D, Mueller L, et al. The incidence of ventilator-associated pneumonia and success in nutrient delivery with gastric versus small intestinal feeding: a randomized clinical trial. Crit Care Med 2000;28:1742-6.

36 Marvin RG, McKinley BA, McQuiggan M, et al. Nonocclusive bowel necrosis occurring in critically ill trauma patients receiving enteral nutrition manifests not reliable clinical signs for early detection. Am J Surg 2000;179:7-12.

37 De Beaux I, Chapman M, Fraser R, et al. Enteral nutrition in the critically ill: a prosepective survey in an australian intensive care unit. Anaesth Intens Care 2001;29:619-22.

38 Heyland DK, Schroter-Noppe D, Drover JW, et al. Nutritional support in the critical care setting: current practice in Canadian ICUs - opportunities for improvement? JPEN J Parenter Enteral Nutr 2003;27:74-83.

39 Verhage AH, van Vliet AC. Clinical practice of nutritional support in Dutch intensive care units: a survey. Eur J Intern Med 2002;13:496-9. 
40 Rubinson L, Diette GB, Song $X$, et al. Low calorie intake is associated with nosocomila bloodstream infections in patients in the medical intensive care unit. Crit Care Med 2004;32:350-7.

41 Ibrahim EH, Mehringer L, Prentice D, et al. Early versus late enteral feeding of mechanically ventilated patients: results of a clinical trial. JPEN J Parenter Enteral Nutr 2002;26:174-81.

42 Heyland DK, Dhaliwal R, Day A, et al. Validation of the Canadian clinical practice guidelines for nutrition support in mechaniscally ventilated, critically ill adult patients: results of a prospective observational study. Crit Care Med 2004;32:2260-6.

43 McClave SA, Lukan JK, Stefater JA, et al. Poor validity of residual volumes as a marker for risk of aspiration in critically ill patients. Crit Care Med 2005;33:324-30.

44 Martin CM, Doig GS, Heyland DK, for the Southwestern Ontario Critical Care Research Network, et al. Multicentre, cluster-randomized clinical trial of algorithms for critical-care enteral and parenteral therapy (ACCEPT). CMA 2004; 170:197-204.

45 Bauer $\mathbf{P}$, Charpentier $\mathrm{C}$, Bouchet $\mathrm{C}$, et al. Parenteral with enteral nutrition in the critically ill. Intens Care Med 2000;26:893-900.

46 Dhaliwal R, Jurewitsch $B$, harrietha $D$, et al. Combination enteral and parenteral nutrition in critically ill patients:harmful or beneficial? A systematic review of the evidence. Intens Care Med 2004;30:1666-71.

47 Van den Berghe G, Wouters P, Weekers F, et al. Intensive insulin therapy in the critically patient. N Engl J Med 2001;345:1359-67.

48 McCowen KC, Friel C, Sternberg J, et al. Hypocaloric total parenteral nutrition: effectiveness in prevention of hypoglycaemia and infectious complications - a randomised clinical trial. Crit Care Med 2000;28:3606-11.

49 Dudrick SJ. Early developments and clinical applications of total parenteral nutrition. JPEN J Parenter Enteral Nutr 2003;27:291-9.

50 Tay SM, Ip-Yam PC, Lim BL, et al. Audit of total parenteral nutrition in an adult surgical intensive care. Ann Acad Med Singapore 2002;31:487-92.

51 Blot DI, Decruyenaere J, Hoste E, et al. Outcome evaluation in ICU patients with catheter-related bloodstream infections. Intens Care Med 2002;20(suppl 1):S11.

52 Aldobo-Pallas T, Garnacho-Montero J, Colon-Pallares C, et al. Evalaution of the outcome of catheter-related bloodstream infections: importance of the pathogen. Intens Care Med 2002;20(suppl 1):S1 1

53 Griffiths RD. Nutrition support in critically ill septic patients. Curr Opin Clin Nutr Metab Care 2003:6:203-10.

54 Suchner U, Katz DP, Furst $P$, et al. Effect of intravenous fat emulsions on lung function in patients with acute respiratory distress syndrome or sepsis. Crit Care Med 2001;29:1569-74.

55 Griffiths RD, Jones C, Palmer TEA. Six month outcome of critically ill patients given glutamine-supplemented parenteral nutrition. Nutrition 1997; 13:295-302.
56 Griffiths RD Allen KD, Andrews FJ, et al. Infection, multiple organ failure, and survival in the intensive care unit: influence of glutamine-supplemented parenteral nutrition on acquired infection. Nutrition 2002; 18:546-52.

57 Novak F, Heyland DK, Avenell A, et al. Glutamine supplementation in serious illness: a systematic review of the evidence. Crit Care Med 2002;30:2022-9.

58 Goeters C, Wenn A, Mertes N, et al. Parenteral L-alanyl-L-glutamine improves 6-month outcome in critically ill patients. Crit Care Med 2002;30:2032-7.

59 Galban C, Montejo JC, Mesejo A, et al. An immune-enhancing diet reduces mortality rate and episodes of bacteremia in septic intensive care unit patients. Crit Care Med 2000;28:643-8.

60 Bower RH, Cerra FB, Bershadsky B, et al. Early enteral administration of a formula (Impact) supplemented with arginine, nucleotides, and fish oil in intensive care unit patients: results of a multicenter, prospective, randomized, clinical trial. Crit Care Med 1995;23:436-49.

61 Atkinson S, Sieffert E, Bihari D. A prospective, randomized, double-blind, controlled clinical trial of enteral immunonutrition in the critically ill. Crit Care Med 1998;26:1 164-72.

62 Heyland DK, Novak F, Drover JW, et al. Should immunonutrition become routine in critically ill patients? A systematic review of the evidence. JAMA $2001 ; 286: 944-53$

63 Braga M, Gianotti L, Nespoli L, et al. Nutritional approach in malnourished surgical patients: a prospective randomized study. Arch Surg 2002;137:174-80

64 Griffiths RD. Specialised nutrition support in critically ill patients. Curr Opin Crit Care 2003:9:249-59.

65 Gadek JE, DeMichele SJ, Karlstad MD, and the Enteral Nutrition in ARDS Study Group, et al. Effect of enteral feeding with eicosapentaenoic acid, $\gamma$ linolenic acid, and antioxidants in patients with acute respiratory distress syndrome. Crit Care Med 1999:27:1409-20.

66 Preiser J-C, Van Gossum A, Berré J, et al. Enteral feeding with a solution enriched with antioxidants vitamins $A, C$, and $E$ enhances the resistance to oxidative stress. Crit Care Med 2000;28:3828-32.

67 Pacht ER, DeMichele SJ, Nelson JL, et al. Enteral nutrition with eicosapentaenoic acid, $\gamma$-linolenic acid, and antioxidants reduces alveolar inflammatory mediators and protein influx in patients with acute respiratory distress syndrome. Crit Care Medicine 2003;31:491-500.

68 Bertolini G, lapichino G, Radrizzani D, et al. Early enteral immunonutrition in patients with severe sepsis: results of an interim analysis of a randomised multicentre clinical trial. Intens Care Med 2003:29:834-40.

\section{ANSWERS}

A. (1) T, (2) T, (3) F, (4) F, (5) F; B. (1) F, (2) T, (3) T, (4) F,

(5) T; C. (1) T, (2) T, (3) F, (4) F, (5) T; D. (1) T, (2) T, (3) F,

(4) $\mathrm{T},(5) \mathrm{T}$

\section{1 th European Forum on Quality Improvement in Health Care}

26-28 April 2006, Prague, Czech Republic

For further information please go to: www.quality.bmjpg.com

Book early to benefit from a discounted delegate rate 